\title{
Similarities and Differences in the Central Nervous System between Different Types of Primary Glaucoma by Multimodal MRI
}

\section{Xuyang Yao}

Peking University First Hospital

Jing Liu

Peking University First Hospital

Yingzi Pan ( $\triangle$ panyingzi99@126.com )

Peking University First Hospital

\section{Xiaoying Wang}

Peking University First Hospital

\section{Chun Zhang}

Peking University Third Hospital

Yan Lu

Beijing shijitan hospital

Yuan Fang

Peking University First Hospital

Yajie Zheng

Peking University First Hospital

Ying Hong

Peking University First Hospital

Yue Wu

Beijing Shijitan Hospital

Queenie Chan

Philips Healthcare

Zhizheng Zhuo

Philips Healthcare

\section{Robert Ritch}

Einhorn Clinical Research Center

Research article

Keywords: , 
Posted Date: October 24th, 2019

DOI: https://doi.org/10.21203/rs.2.16389/v1

License: (c) (i) This work is licensed under a Creative Commons Attribution 4.0 International License. Read Full License 


\section{Similarities and Differences in the Central Nervous System between Different Types of Primary Glaucoma by Multimodal}

\section{MRI}

Xuyang Yao ${ }^{1 \#}$, Jing Liu $^{2 \#}$, Yingzi Pan ${ }^{1 *}$, Xiaoying Wang ${ }^{2 *}$, Chun Zhang ${ }^{3}$, Yan Lu ${ }^{4}$, Yuan Fang $^{1}$, Yajie Zheng ${ }^{1}$, Ying Hong ${ }^{3}$, Yue $\mathrm{Wu}^{4}$, Zhizheng Zhuo ${ }^{5}$, Queenie Chan ${ }^{5}$, Robert Ritch $^{6}$

${ }^{1}$ Department of Ophthalmology, Peking University First Hospital, Beijing, 100034, China

${ }^{2}$ Department of Radiology, Peking University First Hospital, Beijing, 100034, China

${ }^{3}$ Department of Ophthalmology, Peking University Third Hospital, Beijing, 100191, China

${ }^{4}$ Department of Ophthalmology, Beijing Shijitan Hospital, 100038, China

${ }^{5}$ Clinical Science, Philips Healthcare, Beijing, China

${ }^{6}$ Einhorn Clinical Research Center, New York Eye and Ear Infirmary of Mount Sinai, New York, NY 10003

\# These authors contributed equally to this work

*Correspondence: panyingzi99@126.com; cjr.wangxiaoying@vip.163.com

Yingzi Pan

Department of Ophthalmology, Peking University First Hospital, No.8th, Xishiku Street, Xicheng District, Beijing,100034, China

Work Phone: (86) 10 83572748; Tel:(86) 10-13501343177

Email: panyingzi99@126.com

Xiaoying Wang

Department of Radiology, Peking University First Hospital, No.8th, Xishiku Street, Xicheng District, Beijing, 100034, China

Work Phone: (86) 10-83572097; Tel: (86) 10-13511077396

Email: cjr.wangxiaoying@vip.163.com 
Short title: Differences between Primary Glaucoma Patients in the CNS by MRI

\begin{abstract}
:
Background. To investigate the similarities and differences in the structure and function of the central nerve system (CNS) among normal tension glaucoma (NTG), primary open-angle glaucoma with high intraocular pressure (HTG) and primary angle-closure glaucoma (PACG) patients.
\end{abstract}

Methods. Ophthalmic examinations and multimodal magnetic resonance imaging (MRI) were performed. The results of brain functional connectivity (FC) were obtained based on the resting state MRI (rs-fMRI). Voxel-based morphometry (VBM) was applied to analyze structural images to obtain the volume change in the gray matter on 3D T1-weighted imaging (T1WI).

Results. We found that frontal lobe FC increased in all three groups. Temporal lobe FC increased in the NTG group, while the FC of the cingulate gyrus and postcentral gyrus increased in the HTG and PACG groups by rs-fMRI. There were no gray matter volume changes in the NTG, while the gray matter of the frontal gyrus changed in the HTG and PACG. Moreover, the gray matter of the precentral gyrus changed in PACG, and the parietal lobe and supra marginal lobe changed in the HTG. The white matter of the inferior parietal lobe and postcentral gyrus increased, while that in the inferior temporal lobe decreased in the NTG group. The white matter in the HTG and PACG groups showed significant alterations in the medial occipital gyrus.

Conclusions. The NTG group had different manifestations compared with the HTG and PACG groups regarding changes in brain structure and function, suggesting that pathogenic processes might differ between patients with normal-tension and high-tension POAG.

\title{
Background
}

Glaucoma is the most common cause of irreversible blindness worldwide. ${ }^{[1-3]}$ Adult primary glaucoma includes primary angle-closure glaucoma (PACG) and primary 
open-angle glaucoma (POAG). POAG has been historically classified into high-tension glaucoma (HTG), defined as intraocular pressure (IOP) $>21 \mathrm{mmHg}$, and normal-tension glaucoma (NTG), defined as maximal known IOP $\leqslant 21 \mathrm{mmHg}$. These classifications are artificial, as IOP, the most commonly known risk factor for glaucoma, exists as a continuum. For the purposes of clinical research, this terminology has currently been maintained.

Patients with NTG, HTG, and PACG have similar visual field (VF) defects. One of the clinical differences is that both the chamber angles of NTG and HTG are open, while those of PACG is at least partially closed. Another difference is the IOP. The IOP is usually higher than normal in both HTG and PACG but remains within the defined "normal" range in NTG. There has been substantial scientific interest in the differences in optic nerve damage between NTG and HTG. ${ }^{[4-6]}$ In the past decade, several studies have suggested that abnormalities in the CNS are present in POAG, and POAG might be accompanied by descending damage based on neuronal bidirectional trans-synaptic damage ${ }^{[7,8]}$. However, most of the previous studies did not explore differences in the structure and function of the CNS between the HTG and NTG groups.

The development of magnetic resonance imaging (MRI) makes it possible to observe changes in brain structure and function in vivo. This technique has been applied in studies of CNS changes in glaucoma ${ }^{[9-15]}$. The main research techniques of MRI used to evaluate the CNS in glaucoma patients include resting-state functional MRI (rs-fMRI) and voxel-based measurement (VBM) methods. Therefore, we investigated both the functional and structural differences in the CNS of different types of primary glaucoma based on multimodal MRI for the first time, and we hypothesized that there will be differences among these disease types.

\section{Methods}

\section{Subjects}

This study was a cross-sectional study. The research followed the tenets of the Declaration of Helsinki and was approved by the Medical Ethics Review Committee of Peking University First Hospital. All glaucoma patients were recruited from the glaucoma 
specialist outpatient service at Peking University First Hospital, Peking University Third Hospital and Capital Medical University Xuan Wu Hospital from September 2012 to June 2015, based on the clinical diagnostic criteria as described in the supplementary materials. Written informed consent was obtained from all subjects.

The ocular examination included best-corrected visual acuity (BCVA), dilated stereoscopic fundoscopy, Goldmann applanation tonometry, and standard automatic perimetry (SAP) via the Humphrey Swedish Interactive Threshold Algorithm 24-2 test strategy. All participants had undergone at least two prior VF SAP tests. Subjects with fixation loss $>\mathbf{2 0} \%$ or rate of false-positive/negative response $>33 \%$ were excluded from the study based on the exclusion criteria described in the supplementary materials.

\section{Data Acquisition}

All subjects were examined with a $3 \mathrm{~T}$ magnetic resonance scanner (Achieva TX, Philips Medical Systems, Best, the Netherlands) with an 8-channel phased-array head coil. The rs-fMRI data were acquired with the following parameters: repetition time $=2000 \mathrm{~ms}$; echo time $=30 \mathrm{~ms}$; flip angle $=90^{\circ}$; acquisition matrix $=64 \times 64$; field of view $=240 \times 240 \mathrm{~mm}^{2}$; slice thickness $=3 \mathrm{~mm}$ with a $1 \mathrm{~mm}$ gap. Each brain volume comprised 33 axial slices, and each run contained 160 volumes. The parameters of structural MRI used by the 3D MPRAGE sequence included the following parameters: matrix $=256 \times 256 \mathrm{~mm} ; \mathrm{TR}=1830 \mathrm{~ms} ; \mathrm{TE}=4.43 \mathrm{~ms} ; \mathrm{Tl}=1100 \mathrm{~ms} ;$ flip angle $=90^{\circ}$; slice

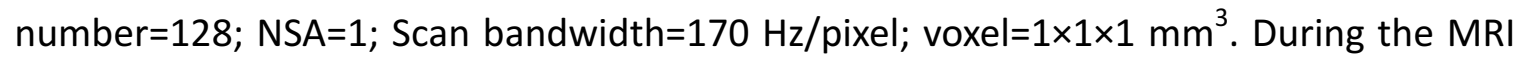
data acquisition, the subjects were instructed to keep their eyes closed, relax their minds but not fall asleep, and remain as motionless as possible.

\section{Data Preprocessing and Analysis}

Data on rs-fMRI processing were obtained via DAPRSF software (Release 2.1; State Key Laboratory of Cognitive Neuroscience and Learning, Beijing Normal University, Beijing, China; available in the public domain at http://restfmri.net/forum/DPARSF). For each subject, the preprocessing steps were as follows:

a. The first 10 volumes were discarded to allow for magnetization equilibration.

b. The remaining volumes were corrected for the acquisition time and realigned to correct for head motion. Subjects with maximum displacement in any direction 
of $>3.0 \mathrm{~mm}$ or head rotation of $>3.0 \mathrm{~mm}$ were excluded from the study. Subjects with incomplete data were also excluded. As a result, the data for 3 NTG patients, 5 PACG patients, and 4 control subjects were excluded, and 88 subjects (25 HTG patients, 22 NTG patients, 20 PACG patients, and 21 controls) were analyzed.

c. The realigned images were then spatially normalized to the Montreal Neurological Institute space and resampled to 3-mm isotropic voxels.

d. For seed-based FC, linear detrending, bandpass filtering, and smoothing were performed before the computation.

\section{Computation of the rs-fMRI parameter and VBM parameters}

The FC strength of the rs-fMRI was defined as the Pearson correlation between the time series within other brain regions and the averaged time series within the seed regions. In this work, BA 18 and BA 19 were selected as the seed regions, and Pearson's correlation coefficient was calculated at the voxel level.

VBM has been used to investigate structural changes in the $\operatorname{brain}^{[16]}$. The preprocessing steps of the VBM analysis are based on SPM software (SPM8; The Welcome Trust Center for Neuroimaging Institute of Neurology, University College London; available in the public domain at http://www.fil.iom.ucl.ac.uk/spm/) and are as follows:

a. Realignment: All T1W images were realigned to the group mean images.

b. Segmentation: The realigned images were segmented with the SPM batch of the New Segment, and gray matter and white matter were obtained for further analysis.

c. Creation of specific templates and normalization: For the control group, specific templates (including gray and white matter templates) were created via the DARTEL method, and then all segmented images were normalized to the corresponding templates.

d. Smoothing: A 4-mm Gaussian kernel was used to smooth the normalized images.

\section{Statistical Analysis}

We compared the anatomic and functional data in the control and glaucoma groups. The baseline characteristics of the groups are shown in Table 1.

For each parameter, an analysis of variance (ANOVA) was first performed to 
determine the differences between the groups, including the 3 subtypes and control groups. Then, multiple comparison analyses $(P<0.05$ with FDR correction and a cluster size of $>20$ voxels) were performed to find the difference between each group of the 3 subtypes and the normal control group. In all statistical analyses, age, gender, and right/left eye were used as covariates.

All data acquisitions were masked. MRI data statistical analyses were performed by two individuals blinded to the diagnostic groups.

\section{RESULTS}

\section{Rest-fMRI}

Compared with the normal controls, the results revealed that temporal lobe FC increased in the NTG group (figure $1 \mathrm{~A}$ ). The cingulate gyrus and postcentral gyrus FC increased in the HTG and PACG groups per rs-fMRI. Frontal lobe FC increased in all three groups (figure $1 \mathrm{~B}$ and figure $1 \mathrm{C}$ ).

\section{VBM}

Compared with the normal control group, there were no gray matter volume changes in the NTG, while the gray matter of the frontal gyrus changed in the HTG and PACG. Moreover, the gray matter of the precentral gyrus was changed in the PACG, and the parietal lobe and supra marginal lobe were changed in the HTG. (figure 2)

Compared with the normal control group, the white matter volume of the inferior parietal lobe and postcentral gyrus increased, while that of the inferior temporal lobe was decreased in the NTG group (figure 3), whereas the white matter of the medial occipital lobe was changed in the HTG and PACG groups (figures 4 and 5). The white matter of the precentral gyrus, middle frontal lobe and temporal lobe also increased in the PACG group (figure 4).

\section{Discussion}

The blood oxygen level-dependent functional MRI (BOLD-fMRI) technique is an imaging method that uses deoxyhemoglobin as an endogenous contrast agent that relies on changes in regional cerebral blood oxygen saturation, which reflects changes in blood volume, blood flow during nerve activity, and hemoglobin oxygenation. This principle can be used to investigate the functions and activities of the $\mathrm{CNS}^{[17]}$, including the 
changes in FC in cerebral areas. To date, BOLD-fMRI has been applied to investigate changes in the CNS in patients suffering from various diseases ${ }^{[18-21]}$.

Characteristic clinical manifestations of glaucoma include an enlarged optic cup, narrowed rim, retinal nerve fiber layer loss, and VF defects. Previous neuroimaging studies have demonstrated that glaucoma might be a neurodegenerative disease, not only referring to an eye disease ${ }^{[1,2]}$, but may also induce functional changes in brain areas $^{[13,22-24]}$. To explore the changes in the CNS of different types of adult primary glaucoma, we investigated the similarities and differences in CNS structure and function in NTG, HTG and PACG patients.

\section{Brain region of functional connectivity}

In the present study, compared with normal controls, frontal lobe FC increased in all three groups. The frontal lobe is involved in optic localization, determining the fixation point and coordinating multiple retinal fields. The frontal lobe also transmits visual information to the oculomotor complex ${ }^{[16]}$. Some prior studies had similar results, although none of these studies analyzed different types of primary glaucoma simultaneously ${ }^{[25,26]}$. Moreover, in our study, when we used BA18 and BA19 (related to superior visual cortex) as the seeds, we found that the connection between the frontal lobe and the occipital lobe significantly increased in all three groups of visual field-defected glaucoma patients, which indicated that the frontal lobe may have a special relationship with the visual pathway. It was recently reported that optic nerve damage leads to disturbed occipitofrontal functional connectivity, and vision recovery seemed to enhance these connections ${ }^{[27,28]}$. These findings demonstrated that frontal lobe activity might be involved in vision impairment in glaucoma. However, how the frontal lobe contributes to vision loss is still weakly defined and needs to be explored. Nevertheless, these results might indicate that glaucoma damage involved not only the visual center but also other parts of the central nervous system.

Notably, there were also some differences between the three groups. Compared with the normal controls, the anterior cingulate cortex and postcentral gyrus FC increased in both the HTG and PACG groups, whereas the temporal lobe FC increased in the NTG group. The functions of the limbic system include emotion, motivation, 
olfaction and long-term memory ${ }^{[29]}$. The cingulate cortex, as part of the limbic lobe, played a crucial role in depression and schizophrenia ${ }^{[30-32]}$ and its connection to the prefrontal cortex is also associated with pleasure ${ }^{[33]}$. The postcentral gyrus is a significant structure in the parietal lobe, which is located in the primary somatosensory cortex. Disorders of this area may cause reduced nociception, and patients may become sensitive. Several studies have reported that glaucoma patients have a high prevalence of anxiety and depression, and the authors considered that the oculopathy, vision loss and side effects of treatment might be the reason for this low quality of life ${ }^{[29,34-37]}$. Therefore, we deduced that both HTG and PACG patients might have CNS damage related to emotional changes. Unfortunately, the reason could not be explained by available studies.

Conversely, temporal lobe abnormalities usually occur in patients with cerebral degenerative disorders, such as Alzheimer's disease ${ }^{[38,39]}$ and frontotemporal dementia. The patients in our NTG group showed a temporal lobe abnormality in the FC parameter of BOLD-fMRI, which was widely reported to be a typical brain disorder manifestation of BOLD-fMRI. Moreover, patients with Alzheimer's disease were reported to have a higher probability of having open-angle glaucoma ${ }^{[40]}$. The present study indicates that our NTG patients might have a neurodegenerative change in the central nervous system, which is similar to the neurodegeneration in senile dementia.

\section{Brain region in voxel-based morphometry analysis}

VBM is an objective method used to evaluate brain volume changes. This method identifies structural changes throughout the brain and permits the recognition of unsuspected potential regions in the brain but is not biased to any particular area ${ }^{[41]}$. VBM has been reported to be used in studies of patients with different kinds of visual field defects and has demonstrated changes in cortical gray and white matter volume ${ }^{[16]}$.

In our study, compared with normal controls, there was no volume change in the gray matter in the NTG group. However, the gray matter of the middle frontal gyrus was significantly changed in the HTG and PACG groups. The middle frontal gyrus is strongly related to the parietal lobule, temporal gyrus, cingulate gyrus, parahippocampal gyrus, angular gyrus, fusiform gyrus and lingual gyrus. As described above, these areas are 
primarily responsible for emotion, behavior, and long-term memory. Therefore, this result is consistent with our previous FC results showing that both HTG and PACG patients might have CNS damage related to negative emotion performance, such as depression.

In this study, no change in white matter volume in the occipital lobe was observed in the NTG group, but the white matter volume of the medial occipital lobe was significantly increased in the PACG group and decreased in the HTG group. Boucard et al. also reported changes in cortical matter density in POAG in the medial occipital cortex ${ }^{[42]}$. There were also several other previous studies that showed visual cortex atrophy and functional impairment in HTG patients, although some of the brain areas were different from each other, possibly because of the different methodology of the MRI scanning and data analysis. Moreover, the different inclusion criteria of the subjects could be another reason for this difference ${ }^{[14,43,44]}$.

However, the changes in the white matter of the occipital lobe were also different between HTG and PACG patients. As mentioned above, the white matter volume of the medial occipital lobe was significantly increased in the PACG group and decreased in the HTG group. Thus far, we do not know the exact reason for this difference, but we speculate that this difference might be due to the different courses of these two diseases. In the case of similar visual field damage, the course of HTG is usually longer than that of PACG. A recent study on early glaucoma showed that before the brain structures start to atrophy, they first become larger and therefore the author reported that the brain might have a temporary compensated stage ${ }^{[45]}$. Therefore, we deduced that at the early stage of PACG, the gray matter volume of the occipital lobe became larger due to brain compensation because of the relatively shorter duration of high IOP attack. However, compared to PACG, HTG patients usually have a long history of moderately elevated IOP and the occipital lobe is decompensated to atrophy. However, this speculation needs to be proven by further study.

\section{Visual pathway in high tension glaucoma and normal tension glaucoma}

In addition to the different brain regions that changed in high tension glaucoma and normal tension glaucoma by using rs-fMRI and VBM analysis, we speculated that there 
might also be some difference in visual pathway injury between these disease types.

The visual pathway can be divided into 2 pathways ${ }^{[46]}$, the magnocellular pathways and parvocellular pathways. After visual information integration and processing in the primary visual cortex (V1), the visual signal is transmitted to the secondary visual cortex (extrastriate cortex) by dorsal and ventral pathways.

The dorsal pathways, which receive signals from the magnocellular pathways, are responsible for spatial information and motional orientation of an object and are mainly located in the parietal lobe, occipital lobe and frontal lobe ${ }^{[47]}$. In our study, we found that frontal lobe FC increased, the gray matter of the frontal gyrus, parietal lobe changed, and the white matter of the medial occipital lobe changed in the HTG and PACG patients, but only frontal lobe FC increased in the NTG patients, which indicated that early and middle stage HTG and PACG patients might show significant changes in the magnocellular pathways, which is consistent with the study by Jiang ${ }^{[48]}$. A previous study also reported that an experimental animal model with high IOP induced early stage glaucoma showed magnocellular pathway disorder, which is also consistent with our results $^{[49]}$.

However, we did not observe occipital lobe changes in our NTG patients via fMRI or VBM analysis in this study. However, we found that temporal FC increased and the white matter of the temporal lobe changed in the NTG group, whereas there were no changes in temporal lobe in the HTG and PACG groups. The ventral pathways are mainly located in the temporal cortex and hippocampus. These results indicated that NTG and HTG had different visual pathway disorders in the early and middle stages. Although all three groups of glaucoma patients showed frontal lobe changes, we deduced that glaucoma and neuropathy patients all had emotion problems, as we mentioned before, which might be the reason for this similarity.

Our findings suggested that although similar visual field deficits are observed in NTG and HTG, these diseases seem to express differential pathophysiology ${ }^{[50]}$. Our speculation is that HTG and PACG might have a different pathological mechanism compared with NTG, and the damage probably results from retinal ganglion cells due to high IOP. In this study, we recruited NTG patients based on the criteria of POAG 
with IOP $\leq 21 \mathrm{mmHg}$, which might include a few NTG patients whose initial IOP was in the low teen values and had the same optic nerve damage mechanism as HTG, although their diagnostic IOP was within the normal range. However, our results showed that our NTG group might also include some patients with other neurodegenerative diseases, such as senile dementia or ischemia.

One limitation of our study is that we had limited cases of all three types of glaucoma patients, which might lead to bias in our results. To verify our observations or to correct some inconsistencies in our data, an investigation with a larger sample size will be necessary to account for glaucoma-related CNS changes ${ }^{[45]}$. Moreover, the consistency and accuracy in the MRI methodology could be the other limitation of our study, which might be influenced by the attention, head movement, or excessive nervousness of the patient. Nevertheless, the main point of our study was to explore the differences in the CNS in primary glaucoma subtypes.

\section{Conclusion}

This study indicated that different types of primary glaucoma with similar visual field damage showed some differences in the structure and function of the brain obtained via multimodal MRI. The NTG group showed some unique manifestations compared to the HTG and PACG groups. Future studies are needed to further explore the structural and functional changes in different types of primary glaucoma.

\footnotetext{
Abbreviations

CNS: central nerve system; NTG: normal tension glaucoma (NTG); HTG: high-tension glaucoma; PACG: primary angle-closure glaucoma; MRI: multimodal magnetic resonance imaging; FC: functional connectivity; rs-fMRI: resting state MRI; VBM: Voxel-based morphometry; T1WI: T1-weighted imaging; POAG: primary open-angle glaucoma; IOP: intraocular pressure; VF: visual field; BCVA: best-corrected visual acuity; SAP: standard automatic perimetry; ANOVA: an analysis of variance; BOLD-fMRI: The blood oxygen level-dependent functional MRI.
} 


\section{Declarations:}

\section{Acknowledgements}

The funding agents of Capital health development and scientific research projects (number: 2011-4021-05) had supported the study including designing, data collection and analysis. All authors declared no conflict of interest.

\section{Authors' contributions}

$\mathrm{XY}, \mathrm{JL}, \mathrm{XW}, \mathrm{YF}$, and YP were responsible for the project design. All authors collected the data. $\mathrm{XY}, \mathrm{JL}$, and $\mathrm{ZZ}$ contributed to the data analysis. $\mathrm{XY}$ made major contributions to writing the manuscript. $\mathrm{JL}, \mathrm{RR}$ and YP revised and edited the manuscript. All authors critically reviewed and approved the final manuscript.

\section{Funding}

This study was supported by grants from Capital health development and scientific research projects (number: 2011-4021-05).

\section{Availability of data and materials}

The data used to support the findings of this study are available from the corresponding author upon request.

\section{Ethics approval and consent to participate}

The study complied with the principles of the Declaration of Helsinki, and was approved by the National Unit of Clinical Trial Ethics Committee, Peking University First Hospital. Written informed consent was obtained from all participants.

\section{Consent for publication}

Not applicable.

\section{Competing interests}

The authors declare that they have no competing interest. 


\section{REFERENCES}

1. Gupta N, Yücel YH. Glaucoma as a neurodegenerative disease. Current Opinion in Ophthalmology. 2007,18(18):110-4.

2. Gupta N, Ang LC, Tilly LND, Bidaisee L, Yücel YH. Human glaucoma and neural degeneration in intracranial optic nerve, lateral geniculate nucleus, and visual cortex. British Journal of Ophthalmology. 2006,90(6):674-8.

3. Broman HAQ, A T. The number of people with glaucoma worldwide in 2010 and 2020. Digest of the World Core Medical Journals. 2006,90(3):262-7.

4. Park JH, Yoo C, Park J, Kim YY. Visual Field Defects in Young Patients With Open-angle Glaucoma: Comparison Between High-tension and Normal-tension Glaucoma. Journal of Glaucoma. 2017,26:1.

5. Pruzan NL, Myers JS. Phenotypic differences in normal vs high tension glaucoma. Journal of neuro-ophthalmology : the official journal of the North American Neuro-Ophthalmology Society. 2015,35 Suppl 1:S4.

6. Hantzschel J, Terai N, Furashova O, Pillunat K, Pillunat LE. Comparison of normal- and high-tension glaucoma: nerve fiber layer and optic nerve head damage. Ophthalmologica Journal international d'ophtalmologie International journal of ophthalmology Zeitschrift fur Augenheilkunde. 2014,231(3):160-5.

7. Qing G, Zhang S, Wang B, Wang N. Functional MRI signal changes in primary visual cortex corresponding to the central normal visual field of patients with primary open-angle glaucoma. Investigative ophthalmology \& visual science. 2010,51(9):4627-34.

8. Chen WW, Wang N, Cai S, Fang Z, Yu M, Wu Q, Tang L, Guo B, Feng Y, Jonas JB, Chen X, Liu X, Gong Q. Structural brain abnormalities in patients with primary open-angle glaucoma: a study with $3 T$ MR imaging. Investigative ophthalmology \& visual science. 2013,54(1):545-54.

9. Nucci C, Mancino R, Martucci A, Bolacchi F, Manenti G, Cedrone C, Culasso F, Floris R, Cerulli L, Garaci FG. 3-T Diffusion tensor imaging of the optic nerve in subjects with glaucoma: correlation with GDx-VCC, HRT-III and Stratus optical coherence tomography findings. British Journal of Ophthalmology. 2012,96(7):976-80.

10. Liu Z, Tian J. Amplitude of low frequency fluctuation in primary open angle glaucoma: A resting state fMRI study. Investigative ophthalmology \& visual science. 2015,56(1):322.

11. Nucci C, Martucci A, Cesareo M, Mancino R, Russo R, Bagetta G, Cerulli L, Garaci FG. Brain involvement in glaucoma: Advanced neuroimaging for understanding and monitoring a new target for therapy. Current opinion in pharmacology. 2012,13(1):128-33.

12. Chen Z, Lin F, Wang J, Li Z, Dai H, Mu K, Ge J, Zhang H. Diffusion tensor magnetic resonance imaging reveals visual pathway damage that correlates with clinical severity in glaucoma. Clinical \& Experimental Ophthalmology. 2013,41(1):43-9.

13. Dai H, Morelli JN, Ai F, Yin D, Hu C, Xu D, Li Y. Resting-state functional MRI: functional connectivity analysis of the visual cortex in primary open-angle glaucoma patients. Human Brain Mapping. 2013,34(10):2455.

14. Chen WW, Wang N, Cai S, Fang Z, Yu M, Wu Q, Tang L, Guo B, Feng Y, Jonas JB. Structural Brain Abnormalities in Patients with Primary Open-Angle Glaucoma: A Study with 3T MR Imaging. Investigative ophthalmology \& visual science. 2013,54(1):545-54.

15. Dai H, Yin D, Hu C, Morelli JN, Hu S, Yan X, Xu D. Whole-brain voxel-based analysis of diffusion tensor MRI parameters in patients with primary open angle glaucoma and correlation with clinical 
glaucoma stage. Neuroradiology. 2013,55(2):233-43.

16. Boucard CC. Changes in cortical grey matter density associated with long-standing retinal visual field defects. Brain. 2009,132(7):1898-906.

17. Song Y, Mu K, Wang J, Lin F, Chen Z, Yan X, Hao Y, Zhu W, Zhang H. Altered spontaneous brain activity in primary open angle glaucoma: a resting-state functional magnetic resonance imaging study. PloS one. 2014,9(2):e89493.

18. Qiu M, Darling WG, Morecraft RJ, Ni CC, Rajendra J, Butler AJ. White matter integrity is a stronger predictor of motor function than BOLD response in patients with stroke. Neurorehabilitation \& Neural Repair. 2010,25(3):275.

19. Patricia Vargas MS, Marie Gaudron MD, Valabrègue R, Eric Bertasi MS, Frédéric Humbert BS, PhD SLM, Yves Samson MD, PhD CRM. Assessment of corticospinal tract (CST) damage in acute stroke patients: Comparison of tract-specific analysis versus segmentation of a CST template. Journal of Magnetic Resonance Imaging. 2013,37(4):836-45.

20. Warbrick T, Fegers-Stollenwerk V, Maximov II, Grinberg F, Shah NJ. Using structural and functional brain imaging to investigate responses to acute thermal pain. Journal of Pain. 2016,17(7):836-44.

21. Toosy AT, Ciccarelli O, Parker GJ, Wheeler-Kingshott CA, Miller DH, Thompson AJ. Characterizing function-structure relationships in the human visual system with functional MRI and diffusion tensor imaging. Neuroimage. 2004,21(4):1452-63.

22. Garaci FG, Bolacchi F, Cerulli A, Melis M, Spanò A, Cedrone C, Floris R, Simonetti G, Nucci C. Optic nerve and optic radiation neurodegeneration in patients with glaucoma: in vivo analysis with 3-T diffusion-tensor MR imaging. Radiology. 2009,252(2):496.

23. Frezzotti P, Giorgio A, Motolese I, Leucio AD, lester M, Motolese E, Federico A, Stefano ND. Structural and Functional Brain Changes beyond Visual System in Patients with Advanced Glaucoma. 2014,9(8):e105931.

24. Dai H, Mu KT, Qi JP, Wang CY, Zhu WZ, Xia LM, Chen ZQ, Zhang H, Ai F, Morelli JN. Assessment of lateral geniculate nucleus atrophy with $3 T \mathrm{MR}$ imaging and correlation with clinical stage of glaucoma. American Journal of Neuroradiology. 2011,32(7):1347-53.

25. Lestak J, Tintera J, Svata Z, Ettler L, Rozsival P. Glaucoma and CNS. Comparison of fMRI results in high tension and normal tension glaucoma. Biomedical Papers of the Medical Faculty of Palacky University I. 2014,158(1):144-53.

26. Yinwei Song KM, Junming Wang, Fuchun Lin, Zhiqi Chen, Xiaoqin Yan, Yonghong Hao, Wenzhen Zhu, Hong Zhang. Altered Spontaneous Brain Activity in Primary Open Angle Glaucoma: A Resting-State Functional Magnetic Resonance Imaging Study. PloS one. 2014,9(2):e89493.

27. Bola M, Gall C, Sabel BA. Disturbed temporal dynamics of brain synchronization in vision loss. Cortex. 2015,67:134-46.

28. Bola M, Gall C, Moewes C, Fedorov A, Hinrichs H, Sabel BA. Brain functional connectivity network breakdown and restoration in blindness. Neurology. 2014,333(6):e556-e.

29. Pache $M$, Flammer J. A sick eye in a sick body? Systemic findings in patients with primary open-angle glaucoma. Survey of Ophthalmology. 2006,51(3):179-212.

30. Hayden BY, Platt ML. Neurons in anterior cingulate cortex multiplex information about reward and action. Journal of Neuroscience. 2010,30(9):3339.

31. Drevets, Wayne C, Savitz, Jonathan, Michael. The Subgenual Anterior Cingulate Cortex in Mood Disorders. Cns Spectrums. 2008,13(8):663-81.

32. Adams R, David AS. Patterns of anterior cingulate activation in schizophrenia: a selective review. 
Neuropsychiatric Disease \& Treatment. 2007,3(1):87-101.

33. Schacter DL, Gilbert DT, Wegner DM. Psychology. Psychology. 2012.

34. Inman WS. EMOTION AND EYE SYMPTOMS. Psychology and Psychotherapy: Theory, Research and Practice. 1921(1).

35. Schoenberg MJ. ROLE OF STATES OF ANXIETY IN THE PATHOGENESIS OF PRIMARY GLAUCOMA. Archives of ophthalmology. 1940,23(1):76-86.

36. Sykes CS. Role of emotion in glaucoma. Diseases of the Nervous System. 1949,10(4):104-7.

37. Yochim BP, Mueller AE, Kane KD, Kahook MY. Prevalence of cognitive impairment, depression, and anxiety symptoms among older adults with glaucoma. Journal of Glaucoma. 2012,21(4):250.

38. Zhang HY, Wang SJ, Xing J, Liu B, Ma ZL, Yang M, Zhang ZJ, Teng GJ. Detection of PCC functional connectivity characteristics in resting-state $\mathrm{fMRI}$ in mild Alzheimer's disease. Behavioural brain research. 2009,197(1):103.

39. Yin H, Yi L, Wang J. Detection of PCC functional connectivity characteristics in subcortical vascular mild cognitive impairment: A resting-state fMRI study. Alzheimers \& Dementia. 2012,8(4):P596-P7.

40. Bayer AU, Ferrari F, Erb C. High occurrence rate of glaucoma among patients with Alzheimer's disease. European Neurology. 2002,47(3):165-8.

41. Astrakas LG, Argyropoulou MI. Shifting from region of interest (ROI) to voxel-based analysis in human brain mapping. Pediatric Radiology. 2010,40(12):1857-67.

42. Boucard CC. Changes in cortical grey matter density associated with long-standing retinal visual field defects. Brain. 2009,132(7):1898.

43. Qing G, Zhang S, Wang B, Wang N. Functional MRI signal changes in primary visual cortex corresponding to the central normal visual field of patients with primary open-angle glaucoma. Investigative ophthalmology \& visual science. 2010,51(9):4627-34.

44. Duncan RO, Sample PA, Bowd C, Weinreb RN, Zangwill LM. Arterial spin labeling fMRI measurements of decreased blood flow in primary visual cortex correlates with decreased visual function in human glaucoma. Vision research. 2012,60(60):51-60.

45. Williams AL, Lackey J, Wizov SS, Chia TM, Gatla S, Moster ML, Sergott R, Spaeth GL, Lai S. Evidence for widespread structural brain changes in glaucoma: a preliminary voxel-based MRI study. Investigative ophthalmology \& visual science. 2013,54(8):5880.

46. Lennie P. Parallel visual pathways: a review. Vision research. 1980,20(7):561-94.

47. Maunsell JH, Nealey TA, DePriest DD. Magnocellular and parvocellular contributions to responses in the middle temporal visual area (MT) of the macaque monkey. The Journal of neuroscience : the official journal of the Society for Neuroscience. 1990,10(10):3323-34.

48. Jiang $M M$, Zhou $Q$, Liu XY, Shi CZ, Chen J, Huang $X H$. Structural and functional brain changes in early- and mid-stage primary open-angle glaucoma using voxel-based morphometry and functional magnetic resonance imaging. Medicine. 2017,96(9):e6139.

49. Ito Y, Shimazawa M, Chen Y-N, Tsuruma K, Yamashima T, Araie M, Hara H. Morphological changes in the visual pathway induced by experimental glaucoma in Japanese monkeys. Experimental Eye Research. 2009,89(2):246-55.

50. Mabuchi F, Sakurada Y, Kashiwagi K, Yamagata Z, lijima H, Tsukahara S. Association between genetic variants associated with vertical cup-to-disc ratio and phenotypic features of primary open-angle glaucoma. Ophthalmology. 2012,119(9):1819-25. 


\section{Figure Legends}

Figure 1 . The results of FC of the 3 subtypes $(P<0.05$ with the FDR correction and a cluster size of $>20$ voxels). A, Temporal lobe FC increased in the NTG group; B, The cingulate gyrus and postcentral gyrus FC increased in the PACG group; $C$, The cingulate gyrus and post-central gyrus FC increased in the HTG group.

Figure 2. The decreased areas of gray matter in the PACG and HTG groups compared to normal controls ( $\mathrm{P}<0.05$ with an FDR correction and a cluster size of $>20$ voxels). $A$, The gray matter of the frontal gyrus and precentral gyrus were changed in the PACG group; B, The gray matter of the frontal gyrus, parietal lobe and supra marginal lobe were changed in the HTG group.

Figure 3. The white matter of the inferior parietal lobe, postcentral gyrus and inferior temporal lobe were changed in the NTG group $(\mathrm{P}<0.05$ with an FDR correction and a cluster size of $>20$ voxels). A, The white matter of the inferior temporal lobe was decreased; B, The white matter of the inferior parietal lobe and postcentral gyrus increased in the NTG group.

Figure 4 . The white matter of the medial occipital lobe, precentral gyrus, middle frontal lobe and temporal lobe were changed in the PACG group $(\mathrm{P}<0.05$ with an FDR correction and a cluster size of $>20$ voxels).

Figure 5. The white matter of the frontal gyrus, parietal lobe, supra marginal lobe changed and medial occipital lobe in the HTG group $(P<0.05$ with an FDR correction and a cluster size of $>20$ voxels). 
Table 1. Baseline Clinical Characteristics

\begin{tabular}{|c|c|c|c|c|}
\hline Characteristics & PACG & HTG & NTG & Controls \\
\hline Age $(y)$ & $59.74 \pm 7.85$ & $53.59 \pm 15.53$ & $54.21 \pm 16.89$ & $62.32 \pm 5.39$ \\
\hline Sex, male/female & $13 / 7$ & $18 / 7$ & $12 / 10$ & $12 / 9$ \\
\hline Visual acuity (OD) & $0.93 \pm 0.22$ & $0.97 \pm 0.14$ & $0.98 \pm 0.1$ & $1.05 \pm 0.09$ \\
\hline Visual acuity (OS) & $0.93 \pm 0.25$ & $0.96 \pm 0.1$ & $0.97 \pm 0.12$ & $1.05 \pm 0.09$ \\
\hline IOP (mmHg) (OD) & $33.9 \pm 18.12$ & $28.04 \pm 3.96$ & $15.4 \pm 1.66$ & $15.92 \pm 1.89$ \\
\hline IOP, mmHg (OS) & $32.8 \pm 17.03$ & $26.64 \pm 4.2$ & $15.4 \pm 1.58$ & $15.9 \pm 1.77$ \\
\hline$M D(d B)$ of poor eye & $-11.55 \pm 6.87$ & $-9.10 \pm 6.44$ & $-9.52 \pm 5.30$ & $-0.32 \pm 0.86$ \\
\hline $\mathrm{MD}(\mathrm{dB})(\mathrm{OU})$ & $-7.27 \pm 4.21$ & $-8.71 \pm 6.61$ & $-5.90 \pm 5.6$ & $-0.16 \pm 0.43$ \\
\hline$C / D$ of poor eye & $0.62 \pm 0.17$ & $0.65 \pm 0.15$ & $0.67 \pm 0.14$ & $0.42 \pm 0.15$ \\
\hline$C / D(O U)$ & $0.34 \pm 0.2$ & $0.51 \pm 0.13$ & $0.57 \pm 0.07$ & $0.21 \pm 0.08$ \\
\hline RNFL of poor eye & $92.64 \pm 19.37$ & $88.36 \pm 12.90$ & $86.30 \pm 13.08$ & $115.05 \pm 11.11$ \\
\hline RNFL (OU) & $90.18 \pm 9.32$ & $82.13 \pm 9.04$ & $83.81 \pm 8.29$ & $110.43 \pm 12.71$ \\
\hline
\end{tabular}




\section{Figures}
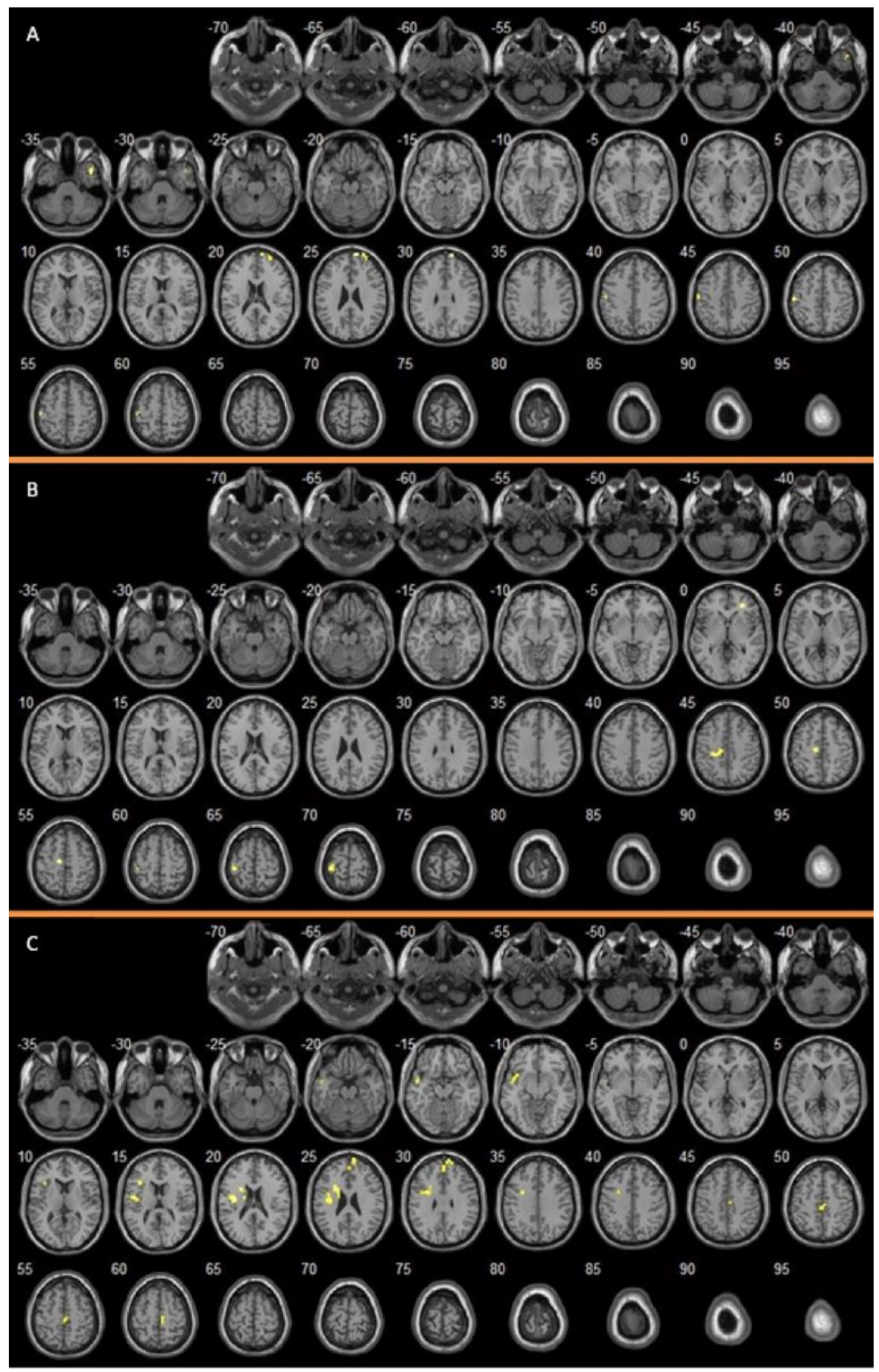

\section{Figure 1}

The results of $F C$ of the 3 subtypes ( $P<0.05$ with the FDR correction and a cluster size of $>20$ voxels). A, Temporal lobe FC increased in the NTG group; B, The cingulate gyrus and postcentral gyrus FC increased in the PACG group; C, The cingulate gyrus and post-central gyrus FC increased in the HTG group. 


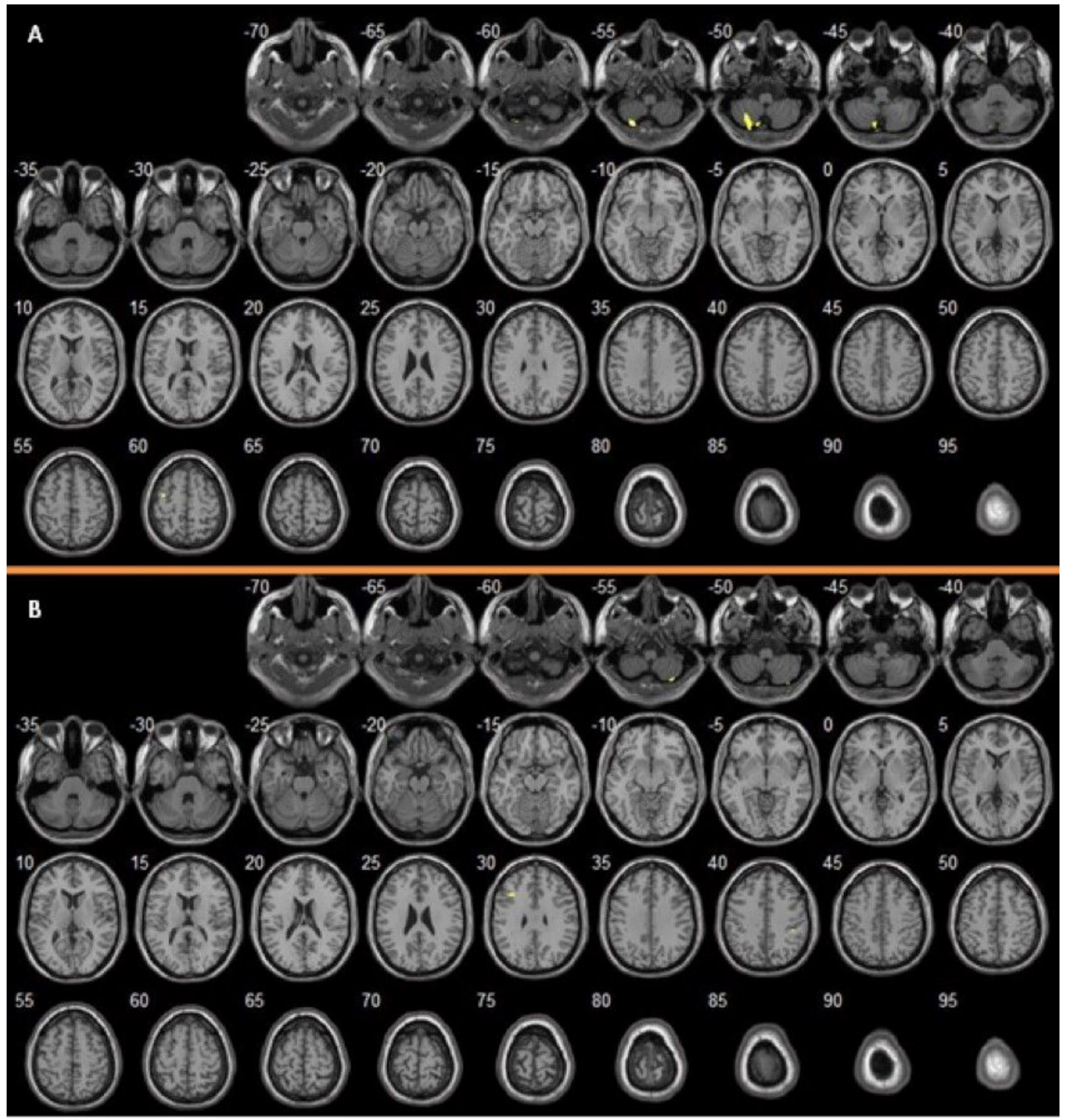

Figure 2

The decreased areas of gray matter in the PACG and HTG groups compared to normal controls $(P<0.05$ with an FDR correction and a cluster size of $>20$ voxels). A, The gray matter of the frontal gyrus and precentral gyrus were changed in the PACG group; B, The gray matter of the frontal gyrus, parietal lobe and supra marginal lobe were changed in the HTG group. 

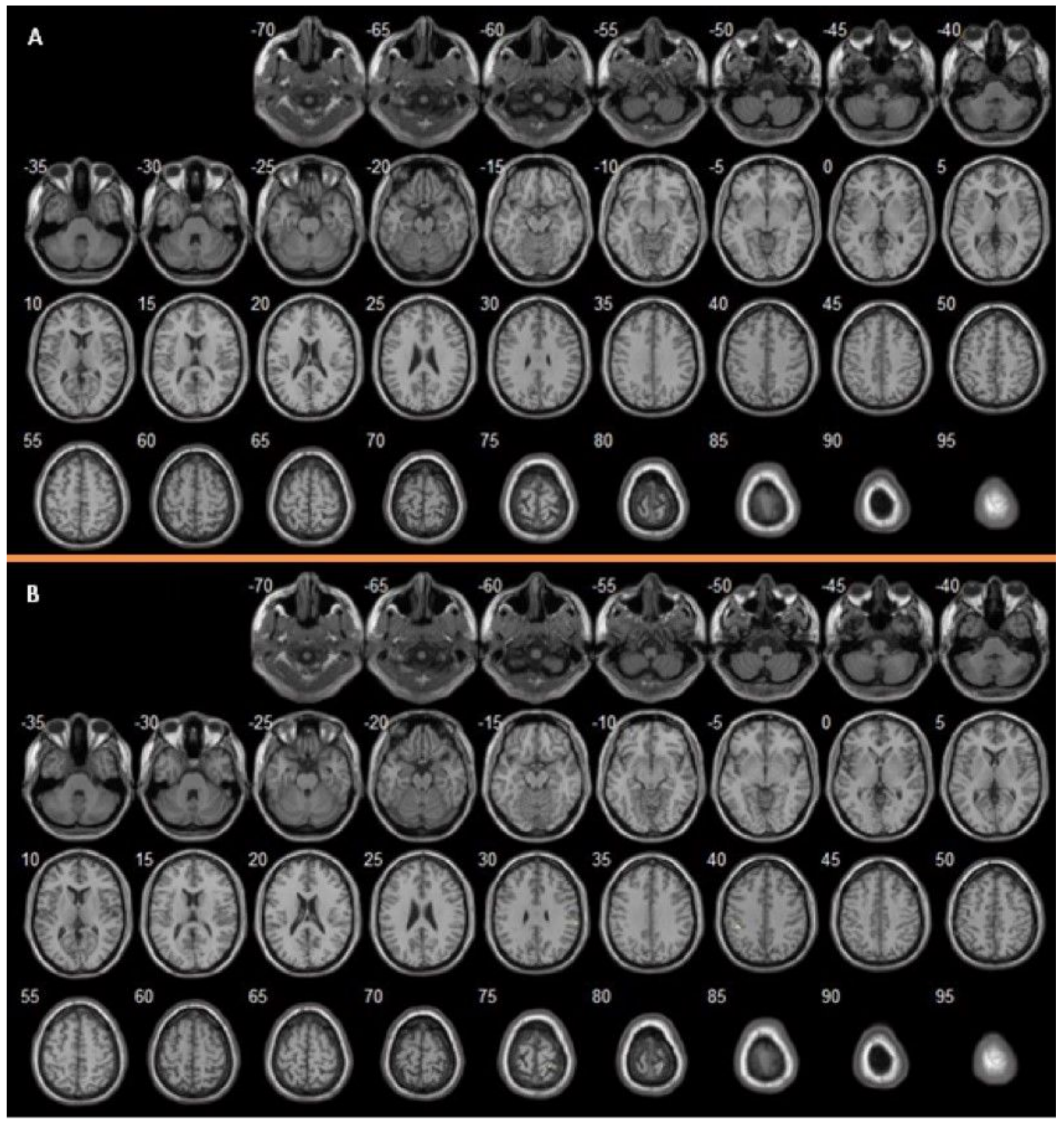

Figure 3

The white matter of the inferior parietal lobe, postcentral gyrus and inferior temporal lobe were changed in the NTG group ( $P<0.05$ with an FDR correction and a cluster size of $>20$ voxels). A, The white matter of the inferior temporal lobe was decreased; $B$, The white matter of the inferior parietal lobe and postcentral gyrus increased in the NTG group. 


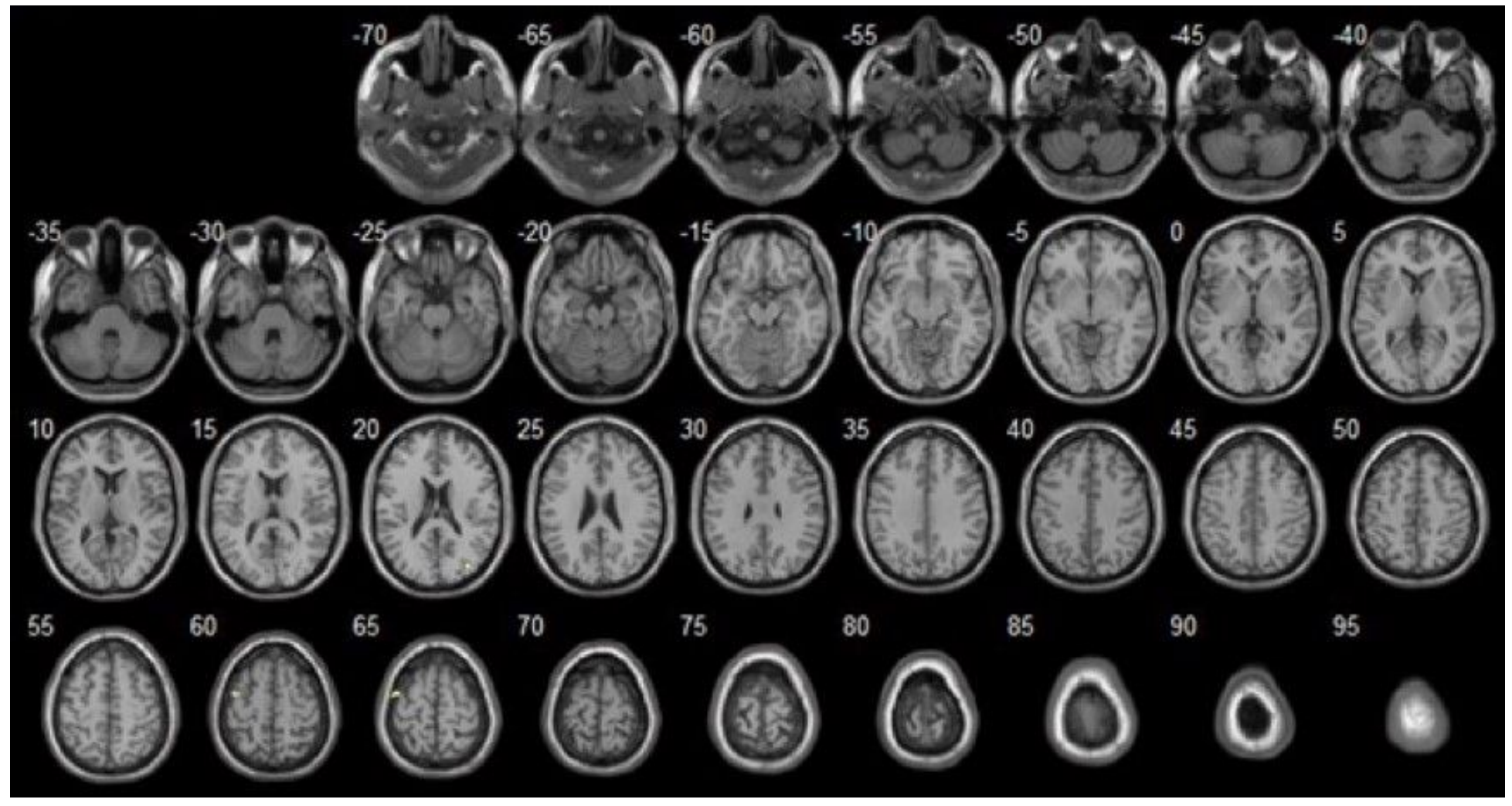

Figure 4

The white matter of the medial occipital lobe, precentral gyrus, middle frontal lobe and temporal lobe were changed in the PACG group ( $\mathrm{P}<0.05$ with an FDR correction and a cluster size of $>20$ voxels).

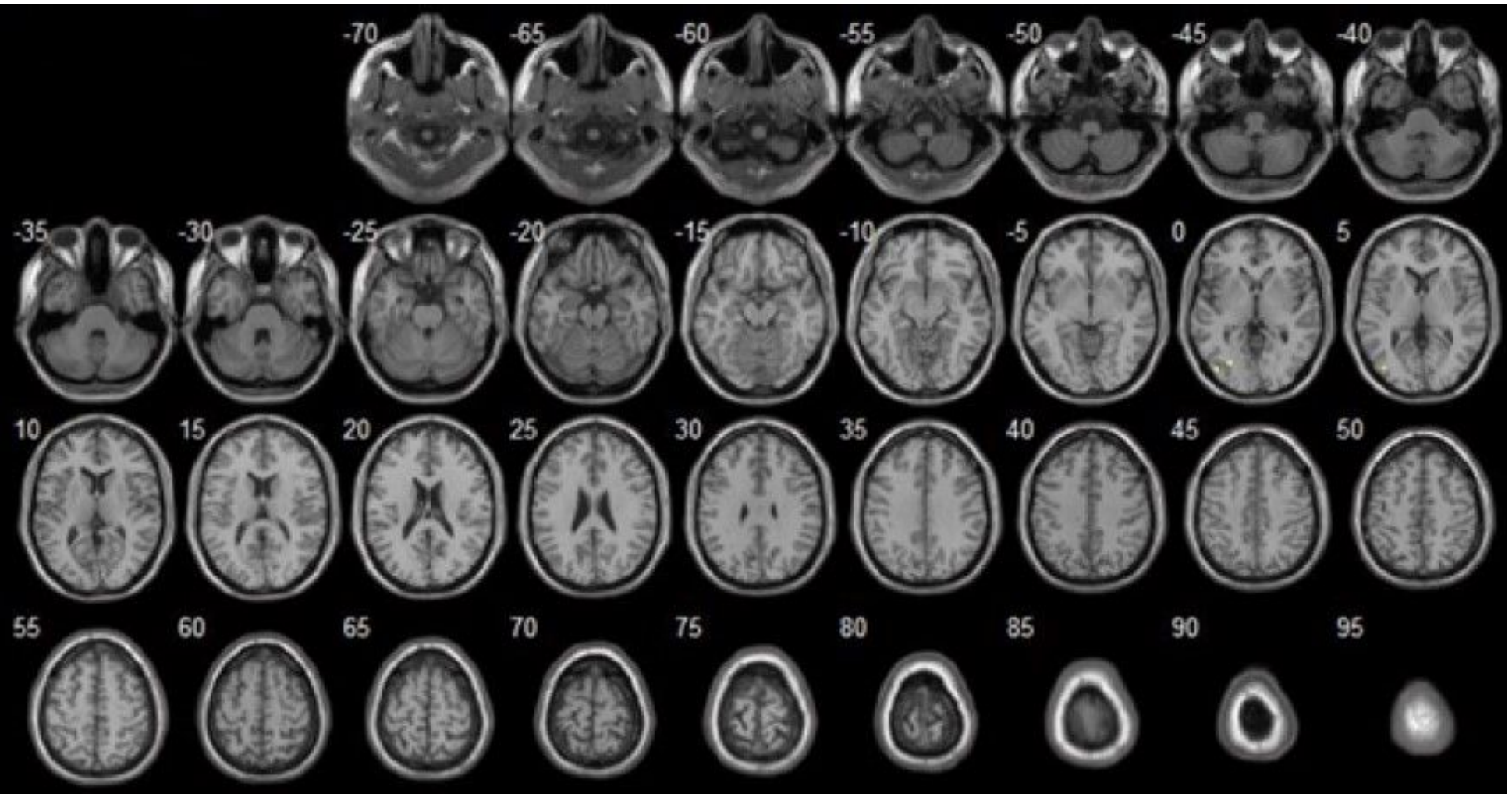

Figure 5 
The white matter of the frontal gyrus, parietal lobe, supra marginal lobe changed and medial occipital lobe in the HTG group ( $P<0.05$ with an FDR correction and a cluster size of $>20$ voxels).

\section{Supplementary Files}

This is a list of supplementary files associated with this preprint. Click to download.

- STROBEchecklist.pdf

- supplement.pdf 